\title{
Comment on
}

\section{"Clouds and the Faint Young Sun Paradox" by Goldblatt and Zahnle (2011)}

\author{
R. Rondanelli ${ }^{1}$ and R. S. Lindzen ${ }^{2}$ \\ ${ }^{1}$ Department of Geophysics, University of Chile, Santiago, Chile \\ ${ }^{2}$ EAPS, MIT, Cambridge, Massachusetts, USA \\ Correspondence to: R. Rondanelli (ronda@dgf.uchile.cl)
}

Received: 30 September 2011 - Published in Clim. Past Discuss.: 21 October 2011

Revised: 24 February 2012 - Accepted: 12 March 2012 - Published: 30 March 2012

\begin{abstract}
Goldblatt and Zahnle (2011) raise a number of issues related to the possibility that cirrus clouds can provide a solution to the faint young sun paradox. Here, we argue that: (1) climates having a lower than present mean surface temperature cannot be discarded as solutions to the faint young sun paradox, (2) the detrainment from deep convective clouds in the tropics is a well-established physical mechanism for the formation of high clouds that have a positive radiative forcing (even if the possible role of these clouds as a negative climate feedback remains controversial) and (3) even if some cloud properties are not mutually consistent with observations in radiative transfer parameterizations, the most relevant consistency (for the purpose of hypothesis testing) is with observations of the cloud radiative forcing. Therefore, we maintain that cirrus clouds, as observed in the current climate and covering a large region of the tropics, can provide a solution to the faint young sun paradox, or at least ease the amount of $\mathrm{CO}_{2}$ or other greenhouse substances needed to provide temperatures above freezing during the Archean.
\end{abstract}

\section{Comments}

We wish to comment on some issues raised in the paper by Goldblatt and Zahnle (2011), hereafter referred to as GZ11, in particular to the criticism of our paper, in which we explored the plausibility of tropical cirrus clouds as a solution to the faint young sun paradox (Rondanelli and Lindzen, 2010a). GZ11 concluded that our solution is not plausible. They base the criticism on the cirrus solution in three main points:
1. Colder temperatures in the Archean are not justified from geological evidence, and therefore solutions to the faint young sun paradox that provide a colder climate than present are not satisfactory; only equal or warmer temperature solutions are acceptable.

2. The mechanism for the increase in the area coverage of cirrus clouds invoked (the iris hypothesis) is controversial and has been questioned. Moreover, even if observed for the present climate, such feedback should have to work for a much colder climate so it would require an unverifiable extrapolation.

3. The cirrus cloud used to represent the tropical cirrus clouds that are sensitive to the surface temperature in the 1-D radiative convective model (one that provides a $50 \mathrm{~W} \mathrm{~m}^{-2}$ radiative forcing in present climate) are unrealistic and represent an end member case of all possible cirrus clouds that can be found to have an impact in the Earth's radiative balance.

The first point relates to the definition of the paradox. A weak version of the paradox would require the possibility of liquid water on some part of the planet's surface. A stronger version requires complete absence of ice on the planet. As we explained in Rondanelli and Lindzen (2010a), we find that cirrus clouds in our model are a plausible solution for the weak version of the paradox; that is, temperature above freezing can indeed be found in some region of the planet, in this case in the tropics, for a reasonable strength of the cloud feedback and for the whole time period. And even for the strong version of the paradox, cirrus clouds provide a large increase in the time span at which mean global 
temperatures are found above freezing. To the extent of our knowledge of the literature, the issue of whether the Earth was hot (that is mean global temperatures of about $55^{\circ} \mathrm{C}$ to $85^{\circ} \mathrm{C}$, at about $3.5 \mathrm{Ga}$; Knauth and Lowe, 2003) or only warm (meaning global temperatures similar to the present) during the Archean has not been settled, given the uncertainties in the oxygen isotopic composition of seawater (see Rosing et al., 2010, and references therein). Kasting et al. (2006), for instance, argue that the isotopic composition of ancient carbonates is not necessarily evidence for a hotter Archean Earth.

On the other hand, the absence of evidence of glaciations cannot be taken to be strong evidence for a significantly warmer or hot Archean Earth, since climate change is typically characterized by changes in the meridional distribution of temperature, rather than uniform changes in temperature. For instance, during most of the last million years, changes in the climate forcing have been only modest with respect to the changes in the solar forcing relevant to the Archean, and yet very different climates have arisen, the latest glacialinterglacial period being just one example. Much warmer climates have occurred, for instance during the Miocene and Eocene, with no significant change in the forcing of either greenhouse gases or solar input compared to changes in the solar forcing we are assuming for the Archean (again, significant, with respect to changes in solar forcing expected to have occurred during the Archean). In order to solve the paradox as usually stated, only warm temperatures are required. Consistency between the weaker solar forcing and a hot planet would constitute a much stronger version of the paradox.

With respect to the second point, the physical mechanism invoked is secondary to the original question we attempt to answer. That is, whether cirrus clouds such as those observed in present climate can provide enough cloud greenhouse warming so as to produce surface temperatures above freezing for a reduced solar insolation, such as found in the Archean, without resorting to other greenhouse substances. The answer to this question appears to be yes. To be sure, the iris hypothesis can not be discarded as a mechanism operating in the tropics. The challenges to the original observational analysis quoted by GZ11 were properly answered (see e.g. Lindzen et al., 2002; Chou and Lindzen, 2005) and evidence for a negative correlation between area of cirrus clouds and sea surface temperature from independent data and researchers has appeared since then (see Rondanelli and Lindzen, 2010b, for the references). Nevertheless, the radiative effect associated with the iris effect, and therefore the magnitude of the cloud feedback remains to be established. However, the point of our original paper was simply to ask the question of how far can the cirrus cloud forcing go toward solving the faint young sun paradox, regardless of what mechanism produced the cirrus clouds.

Perhaps the most important point is the one related to the realism of the cirrus cloud used as a surrogate for those clouds sensitive to the surface temperature in the model. We chose the cloud not arbitrarily (and certainly not to optimize the warming as suggested by GZ11), but rather to be consistent with the available estimations for the cloud radiative forcing obtained by Choi and Ho (2006). This consistency with the radiative forcing does not imply consistency with the physical properties of the clouds due to inherent difficulties in the modeling of radiative transfer in cirrus clouds (e.g. Baran, 2009). As suggested by one of the reviewers, it is also possible that the implicit representation of the effect of low and middle clouds in our original solution (through the surface albedo) may result in some of the cirrus properties being unrealistic.

Nevertheless, for the purpose of radiative transfer, different empirical parameterizations for the visible optical depth $\tau$ can be obtained from in-situ observations of cirrus clouds (e.g. Heymsfield et al., 2003). In these parameterizations, $\tau$ is usually a function of the ice water path and the effective particle radius. If one fixes the ice water path and the effective particle radius, different values for $\tau$ (up to a factor of 2) can be obtained. This, in part, explains the approach taken in Rondanelli and Lindzen (2010a); instead of providing accurate inputs to the 1-D model for every cloud property (that is, observed particle radius, optical depth and ice water path), the idea was to provide a cloud radiative forcing that was consistent with observations. By attempting to obtain a realistic cloud radiative forcing, the effective particle radius (in the parameterization used in our radiative code) lies in the upper tail of the observed particle size distribution of thin cirrus clouds (Kahn et al., 2008). Nevertheless, as shown by Choi and Ho (2006) and others (e.g. Kubar et al., 2007), cirrus clouds having radiative forcing higher than $50 \mathrm{~W} \mathrm{~m}^{-2}$ can cover about $16 \%$ of the tropics. Models allow for net radiative forcings higher than $50 \mathrm{~W} \mathrm{~m}^{-2}$ for a large range of cloud microphysical parameters using a standard tropical atmosphere (e.g. Hong et al., 2009). Therefore, the proposition that the cirrus clouds used were "end member" cases might be true for a particular radiative model or for a particular cloud property; in our case cloud radiative forcing, visible optical depth, height of the cloud and ice water path, all lie well within the observed range of parameters.

\section{Conclusions}

Many interesting ideas arise as one starts considering clouds as a possible solution to the paradox. As both Rondanelli and Lindzen (2010a) and GZ11 found, clouds modify the impact of other greenhouse substances, for instance, by increasing the amount of $\mathrm{CO}_{2}$ required to solve the paradox from what was calculated in simple 1-D models with no clouds. Similarly, as we show in our paper, clouds also modify the strength of the water vapor feedback and the strength of the hydrological cycle by altering the surface heat balance (presumably having an impact in the feedback mediated through 
the inorganic carbon cycle). Since the work of Rossow et al. (1982), it appears that clouds have been omitted from consideration, not so much because of their irrelevance, but rather because of the difficulty constraining their behavior. We agree with GZ11 in the need of including clouds in subsequent efforts to model the problem; we disagree with their assessment on the plausibility of the solution we proposed.

As commented by the reviewers, 2-D and 3-D models can be useful to explore the mutual interaction of cloud feedbacks with other processes in the climate system that might be relevant for the faint young sun paradox, for instance, the meridional heat transport and the ice-albedo feedback. However, without the guidance of simpler models that capture the relevant physics, it is easy to increase the complexity of the simulations without necessarily increasing understanding, especially given the still very coarse treatment of clouds in general circulation models.

Acknowledgements. We appreciate the reviews by J. Kasting and I. Halevy and also the editorial support by A. Paul. R. R. acknowledges support from FONDECYT through grant 11100393.

Edited by: A. Paul

\section{References}

Baran, A.: A review of the light scattering properties of cirrus, J. Quant. Spectrosc. Ra., 110, 1239-1260, 2009.

Choi, Y.-S. and Ho, C.-H., Radiative effect of cirrus with different optical properties over the tropics in MODIS and CERES observations, Geophys. Res. Lett., 33, L21811, doi:10.1029/2006GL027403, 2006.

Chou, M. and Lindzen, R.: Comments on "Examination of the Decadal Tropical Mean ERBS Nonscanner Radiation Data for the Iris Hypothesis", J. Climate, 18, 2123-2127, 2005.

Goldblatt, C. and Zahnle, K. J.: Clouds and the Faint Young Sun Paradox, Clim. Past, 7, 203-220, doi:10.5194/cp-7-203-2011, 2011.
Heymsfield, A., Matrosov, S., and Baum, B.: Ice Water PathOptical Depth Relationships for Cirrus and Deep Stratiform Ice Cloud Layers, J. Appl. Meteorol., 42, 1369-1390, 2003.

Hong, G., Yang, P., Baum, B., Heymsfield, A., and Xu, K.: Parameterization of Shortwave and Longwave Radiative Properties of Ice Clouds for Use in Climate Models, J. Climate, 22, 62876312, 2009.

Kahn, B. H., Liang, C. K., Eldering, A., Gettelman, A., Yue, Q., and Liou, K. N.: Tropical thin cirrus and relative humidity observed by the Atmospheric Infrared Sounder, Atmos. Chem. Phys., 8, 1501-1518, doi:10.5194/acp-8-1501-2008, 2008.

Kasting, J., Howard, M., Wallmann, K., Veizer, J., Shields, G., and Jaffrés, J.: Paleoclimates, ocean depth, and the oxygen isotopic composition of seawater, Earth Planet. Sc. Lett., 252, 82-93, 2006.

Knauth, L. and Lowe, D.: High Archean climatic temperature inferred from oxygen isotope geochemistry of cherts in the $3.5 \mathrm{Ga}$ Swaziland Supergroup, South Africa, B. Geol. Soc. Am., 115, 566-580, 2003.

Kubar, T., Hartmann, D., and Wood, R.: Radiative and Convective Driving of Tropical High Clouds, J. Climate, 20, 5510-5526, 2007.

Lindzen, R., Chou, M., and Hou, A.: Comment on "No Evidence for Iris", B. Am. Meteorol. Soc., 83, 1345-1349, 2002.

Rondanelli, R. and Lindzen, R.: Can thin cirrus clouds in the tropics provide a solution to the faint young Sun paradox?, J. Geophys. Res., 115, D02108, doi:10.1029/2009JD012050, 2010a.

Rondanelli, R. and Lindzen, R.: Comment on "Variations of tropical upper tropospheric clouds with sea surface temperature and implications for radiative effects" by $\mathrm{H}$. Su et al., J. Geophys. Res., 115, D06202, doi:10.1029/2008JD011189, 2010b.

Rosing, M., Bird, D., Sleep, N., and Bjerrum, C.: No climate paradox under the faint early Sun, Nature, 464, 744-747, 2010.

Rossow, W., Henderson-Sellers, A., and Weinreich, S.: Cloud Feedback: A Stabilizing Effect for the Early Earth?, Science, 217, 1245-1247, 1982. 